\title{
Characterization of the Anthelmintic Activity of Murraya koenigii (Linn.)
}

\author{
Sujith $\mathrm{S}^{*}$, Priya $\mathrm{MN}^{2}$, Deepa $\mathrm{CK}^{3}$, Usha PTA ${ }^{4}$
}

\section{Sujith $\mathrm{S}^{*}$, Priya $\mathrm{MN}^{2}$, Deepa $\mathrm{CK}^{3}$, Usha PTA}

${ }^{1}$ Assistant Professor, Department of Veterinary Pharmacology and Toxicology, College of Veterinary and Animal Sciences, Mannuthy, INDIA.

${ }^{2}$ Assistant Professor, Department of Veterinary Parasitology, College of Veterinary and Animal Sciences, Mannuthy, Kerala, INDIA. ${ }^{3}$ Assistant Professor, Department of Veterinary Parasitology, College of Veterinary and Animal Sciences, Pookode, Kerala, INDIA.

${ }^{4}$ Professor and Head, Department of Veterinary Pharmacology and Toxicology, College of Veterinary and Animal Sciences, Mannuthy, Kerala, INDIA.

Correspondence

Mr. Sujith S

Assistant Professor, Department of Veterinary Pharmacology and Toxicology, College of Veterinary and Animal Sciences, Mannuthy, Kerala, INDIA.

Phone no : +91-9539511273

E-mail: sujith@kvasu.ac.in

History

- Submission Date: 13-09-2017;

- Review completed: 02-11-2017;

- Accepted Date: 19-04-2018

DOI : 10.5530/pj.2018.6s.19

Article Available online

http://www.phcogj.com/v10/i6s

\section{Copyright}

(C) 2018 Phcog.Net. This is an openaccess article distributed under the terms of the Creative Commons Attribution 4.0 International license.

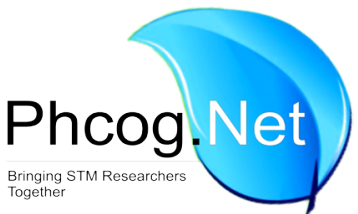

\begin{abstract}
Objective: To identify the most potent sub fractions(s) of the different extracts of the leaves of Murraya koenigii for the anthelmintic property. Methods: The dried leaves were subjected to soxhlet extraction using methanol, fractionated using n-hexane, chloroform, n-butanol and water. Preliminary phytochemical analysis was done using standard techniques. The potent fractions were subjected to TLC and the appropriate solvent was selected for flash chromatographic separation of the extract. The sub fractions were tested for their anthelmintic activity in vitro using egg hatch assay and larval motility assay on Haemonchus contortus eggs and the most potent fraction was found out. Results: Phytochemical analysis revealed the presence of phenolic, tannins and saponins in all extracts and the effect of the extracts could be due to these components. On TLC, toluene: ethyl acetate in 9:1 ratio was found to be the best mobile phase for hexane and chloroform fractions whereas cyclohexane: ethyl acetate at 6:4 was found suitable for butanol fraction. Of the sub fractions (SF), SF 3 and 11 of chloroform fraction showed better ovicidal activity whereas SF 2,6,7,32 and 37 showed best larvicidal activity. The larvae that were used for testing the larvicidal activity, were found to be sluggishly motile after half an hour incubation with the extract and were progressively dead on a dose dependent manner. Conclusion: The chloroform extract of Murraya koenigii and its sub fractions 2, 3,6, 7, 11, 32 and 37 possessed good anthelmintic activity and the isolation of active molecules is necessary for development of a novel anthelmintic.

Key words: Anthelmintic, Murraya koenigii, Haemonchus contortus, Egg hatch assay, Larval motility assay.
\end{abstract}

\section{INTRODUCTION}

Gastrointestinal helminthosis pose a great threat to livestock industry affecting the productivity of ruminants. They affect the production and reproduction parameters by interfering with nutrient absorption and causing intestinal pathologies. ${ }^{1}$ Most of the present day Anthelmintics viz, benzimidazoles, macro cyclic lactones and imidazothiazoles show development of resistance worldwide, which may be for a single class or multi drug resistance and hence an alternate must be found out. Herbal agents form a better solution as they are nontoxic and economically viable and so research on the anthelmintic activity of medicinal plants has got a great drive. ${ }^{2}$

Murraya koenigii, commonly known as curry leaf tree, belongs to the family rutacea and is seen all over India, Srilanka and South East Asia. It is well known for its medicinal properties like antibacterial, antifungal, cytotoxic, antidiarrheal, anti-inflammatory and cytotoxicity. ${ }^{3}$ Anthelmintic activity of Murraya koenigii extracts has been assessed in Pheretima posthuman ${ }^{4,5}$ but literature is scarce on the ovicidal and larvicidal activity against the nematodes. Hence the present work has been undertaken to assess the ovicidal and larvicidal activity of different extracts of $M$. koenigii on $\mathrm{L}_{3}$ larvae of Haemonchus contortus.

\section{MATERIALS AND METHODS}

\section{Plant Material}

The leaves of Murraya koenigii was collected from different parts of the district of Wayanad, identified and authenticated by a Botanist at MSSRF, Kalpetta. It was dried under shade and pulverized. They were extracted using methanol in soxhlet extraction apparatus, dried using a rotary vacuum evaporator and stored under refrigeration till further use. The aqueous extract was taken as a decoction. 50\% hydro-alcoholic extract was taken using methanol: water; 1:1 in a soxhlet extraction apparatus and both were dried and stored under refrigeration till further use.

\section{Fractionation of the extract}

The methanolic extract was further fractionated in a separating funnel by taking solvents in order of increasing polarity viz, hexane, chlo- 
roform, n-butanol and water. They were dried using rotary vacuum evaporator and stored under refrigeration till further use.

\section{Thin Layer chromatography}

The extracts that showed good results with the ovicidal and larvicidal activity were subjected to thin layer chromatography using combination of solvents. The fractions showing best results were selected for doing flash chromatography. The sub fractions were also subjected for ovicidal, and larvicidal activity.

\section{Phytochemical analysis}

The extract as well as the fractions was analyzed qualitatively for various phytochemical constituents. ${ }^{6}$

\section{Assessment of the anthelmintic activity Egg hatch assay}

Fresh ova were collected from fecal sample of goat infested with Haemonchus contortus, concentrated by centrifugation, washed with distilled water and used.The extracts as well as the fractions were diluted to concentrations of $100,50,25,12.5,6.25,3.125$ and $1.5625 \mathrm{mg} / \mathrm{ml}$ in a total volume of $0.5 \mathrm{ml}$. Albendazole and Ivermectin were used as positive control @ 1 and $0.5 \mathrm{mg} / \mathrm{ml}$ respectively, whereas distilled water served as negative control. About 50eggs $/ 0.5 \mathrm{ml}$ distilled water were counted and taken in marked 6- well tissue culture plates and were added with $0.5 \mathrm{ml}$ of the extract as described earlier. The effective concentration of the drug in each petriplate was thus reduced to $50,25,12.5,6.25,3.125$, 1.5625 and $0.78125 \mathrm{mg} / \mathrm{ml}$. The sub fractions obtained from flash chromatography were diluted @ 500, 250, 125, 62.5, 36.125 and $15.6 \mu \mathrm{g} / \mathrm{ml}$. The culture plates were incubated for $48 \mathrm{hrs}$ at $27^{\circ} \mathrm{C}$. The experiment was done in triplicates for each concentration. Hatched larvae (dead or alive) and unhatched eggs were counted under dissection microscope (magnification $40 \mathrm{X})^{7}$ The lowest concentration that produced mortality in $50 \%$ of ova was taken as Minimum Inhibitory concentration.

\section{Larval Motility Assay}

Five gram of dung from goats infested with Haemonchus contortus were incubated at room temperature with adequate humidity in dark for 10 days to get L3 larvae. The larvae were washed out into petri plates using distilled water. The larvicidal activity was done as per the procedure of Rahman et al., $2011^{8}$ with minor modifications. Approximately 100 motile larvae were collected in $100 \mu \mathrm{L}$ water into which equal quantity of extract diluted in distilled water were added. Extracts were prepared as described for egg hatch assay. The loss of motility of the larvae was checked every 15 min and the Percentage larvae found non-motile/ dead were calculated. The lowest concentration that produced mortality in $50 \%$ of larvae was taken as Minimum Inhibitory concentration.

\section{Assessment of the acute oral toxicity}

The acute oral toxicity of all the extracts tested were done in rats at the dose of $2000 \mathrm{mg} / \mathrm{kg}$ as per OECD guidelines $420{ }^{9}$

\section{RESULTS AND DISCUSSION}

\section{Phytochemical analysis.}

The results of phytochemical analysis is presented in Table 1. Phytochemical analysis revealed the presence of tannins and flavonoids in all the extracts and fractions whereas steroids were absent in all the extracts. Saponins were detected only in the aqueous extract.

\section{Solvent selection and Column chromatographic separation of different extracts}

On thin layer chromatography, the hexane and chloroform extracts showed maximum separation using Toluene: Ethyl acetate in 9:1 ratio,
Table 1: Phytochemical constituents of extracts of Murraya koenigii leaf.

\begin{tabular}{|c|c|c|c|c|c|c|c|}
\hline \multirow[t]{2}{*}{ Constituents } & \multicolumn{7}{|c|}{ Murraya koenigii leaf } \\
\hline & $\begin{array}{l}\frac{n}{\partial} \\
\frac{0}{d} \\
\frac{0}{\sigma} \\
\frac{0}{\alpha}\end{array}$ & 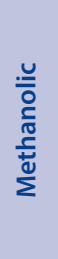 & 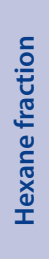 & 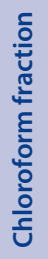 & 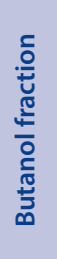 & 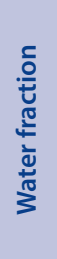 & 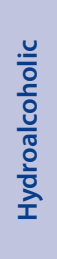 \\
\hline Phenolics & + & + & - & + & + & + & + \\
\hline Alkaloids & - & + & + & + & - & - & + \\
\hline Steroids & - & - & - & - & - & - & - \\
\hline Glycosides & + & + & + & + & - & - & + \\
\hline Tannins & + & + & + & + & + & + & + \\
\hline Terpenes & - & + & + & + & - & - & - \\
\hline Saponins & + & - & - & - & - & - & - \\
\hline Flavonoids & + & + & + & + & + & + & + \\
\hline
\end{tabular}

and butanol extract with cyclohexane: ethyl acetate at 6:4. Since the chloroform extract showed best results, it was further fractionated with the solvent. The fractionation using flash chromatography yielded 42 fractions which were subjected to further testing.

\section{Assessment of anthelmintic activity Egg Hatch Assay}

From the results represented in Figure 1, it is seen that both the methanolic as well as aqueous extracts showed ovicidal activity and of the fractions, hexane fraction showed lowest MIC (Figure2). But when the fractions were sub fractionated, the Chloroform sub fractions 3 and 11 showed maximum potency with MIC as low as $15.6 \mu \mathrm{g} / \mathrm{ml}$. The activity of the chloroform fraction was due to the action of SF 3 and 11 where as that of hexane is due to SF 11 and 19, and butanol fraction by SF 18 and 21 . Further characterization of the sub fractions 3 and 11 of chloroform fraction using chromatography and identification of the molecule by LCMS can give a lead to a novel anthelmintic.

\section{Larval Motility Assay}

The results (Figure2) indicate that chloroform fraction and its sub fractions 6, 3, 32 were the most potent with lowest MIC's whereas butanol and hexane sub fractions had lower potency with MIC of $3.25 \mathrm{mg} / \mathrm{ml}$. However the chloroform sub fraction 32, butanol sub fractions 2, 13 and 14 were of equal potency showing MIC of $36.25 \mu \mathrm{g} / \mathrm{ml}$. The chloroform fraction again proves to be a good anthelmintic and separation of its fractions needs to be done.

\section{Acute oral toxicity}

No mortality was detected in all groups of animals treated with the extract. Also no untoward clinical signs were noticed in any of the animals treated with the extract during the entire period of observation.

\section{DISCUSSION}

Screening of molecules for anthelmintic activity is mainly done using invitro and invivo techniques. Invitro techniques include (i) egg hatch 


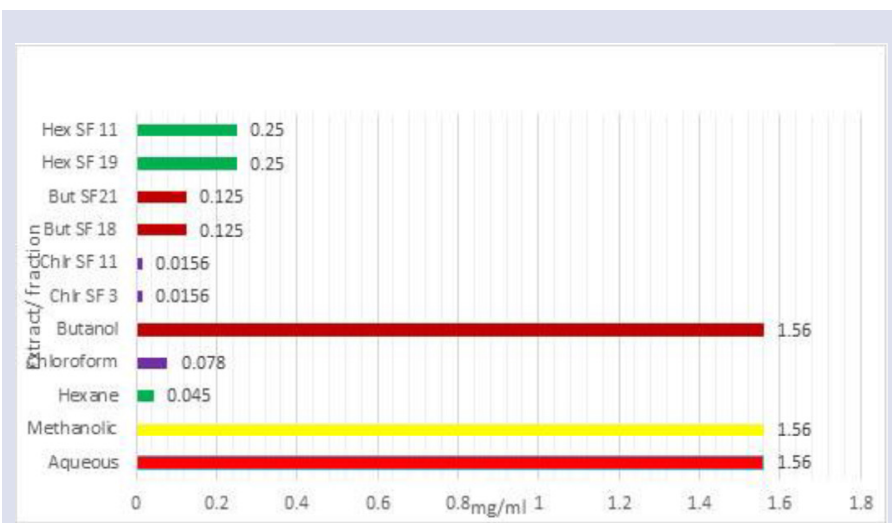

Figure 1: Minimum Inhibitory Concentration of various extracts and sub fractions of Curry leaf on hatch of Haemocnchus contortus eggs. assay estimating the ovicidal activity, (ii) effect on the larvae assessed by larval motility assay, larval development assay and larval migration inhibition assay ${ }^{10,11}$ (iii) adulticidal activity assessed by the effect on motility, paralysis and death of the worms. ${ }^{12,13}$ Since the anthelmintic activity of a broad spectrum agents can be on any of the three stages viz, ova, larvae or adult or more than one of these, screening in all the three stages will provide the exact mechanism of action of the molecule.

The phytochemical analysis (Table 1) revealed presence of tannins, flavonoids, terpenes, phenolic compounds in almost all the extracts. Tannins will affect the energy metabolism of the parasites, may affect the integrity of the cuticle and also impair feeding and reproduction, mainly by their effect on proteins. ${ }^{1,7}$ Tannins can inhibit oxidative phosphorylation, thus decrease metabolism and availability of energy leading to death of the larvae. ${ }^{14}$ Tannin containing plants are reported to provide a method of natural control of helminth infections. ${ }^{15,16}$ Continuous feeding of plants like Sericea lespedeza rich in condensed tannins reduced the egg production, hatchability, larval development and a concomitant reduction in pasture contamination by gastrointestinal nematodes. ${ }^{17}$ Saponins affect the cell wall integrity interact with the collagen of the cuticle where by the cell will lose electrolytes and chemicals and thus the circular damage will be sufficient for the death of the parasite. ${ }^{18,19}$ The presence of flavonoids in the plant extracts affect the moulting as well as the survival of various larvae and potentiates the activity of various other drugs, chemicals etc..$^{20}$

From the results of the study, it is seen that the sub fractions (SF), SF 3 and 11 of chloroform fraction showed maximum ovicidal activity whereas SF 2,6,7,32 and 37 showed maximum larvicidal activity. The ova were found to be disintegrated in the higher doses whereas the hatching was inhibited at lower doses. The results suggest that the effect on the ova can be on the shell, where in the ova becomes disintegrated. The larvae that were used for testing the larvicidal activity, were found to be sluggishly motile after half an hour incubation with the extract and were progressively dead on a dose dependent manner. It can be interpreted that the extract could be affecting the energy production or utilization process of the larvae. Identification of the molecule present in the sub fractions will provide with a novel anthelmintic with a broad spectrum of activity.

\section{CONCLUSION}

From the results of the study, it could be concluded that the chloroform fraction of the methanolic extract of leaves of $M$. koenigii and its subfractions $2,3,6,7,11,32$ and 37 possessed good anthelmintic activ-

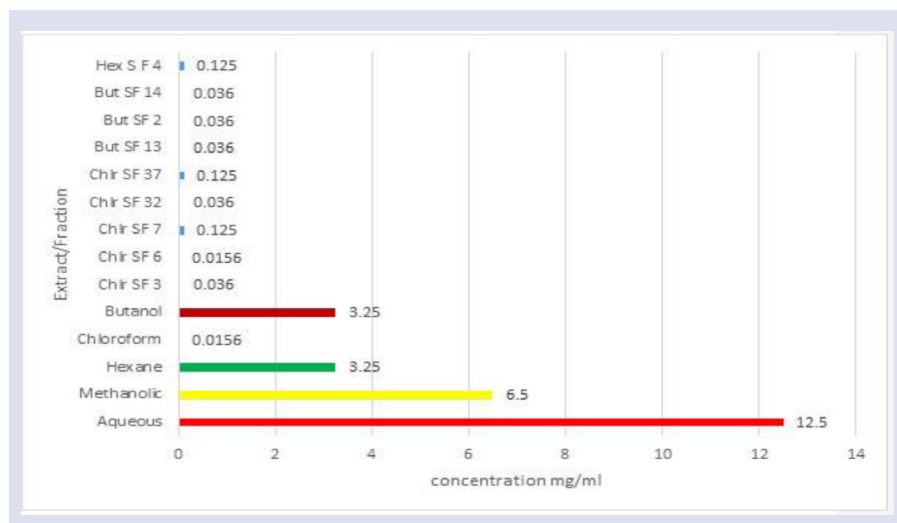

Figure 2: Effect of various extracts on the larval survival.

ity as evidenced from the results of egg hatch assay and larval motility assay in Haemonchus contortus worms. Further isolation of the active compounds can provide a lead for the development of a novel and safe anthelmintic.

\section{ACKNOWLEDGEMENT}

The authors acknowledge Kerala State Council for Science, Technology and Environment (KSCSTE) for the financial support and Kerala Veterinary and Animal Sciences University, Kerala, India for the facilities provided.

\section{CONFLICT OF INTEREST}

The authors declare no conflict of interest.

\section{REFERENCES}

1. Bachaya HA, IqbalZ, Khan MN, Sindhu Z, JabbarS. Anthelmintic activity of ziziphus nummularia (bark) and Acacia Nilotic a (fruit) against Trichostronglyloid nematodes of sheep. J Ethnopharmacol. 2009;123(2):325-9.

2. Iqbal Z, Lateef $M$, Ashraf $M$, Jabbar A.Anthelmintic activity of Artemisia brevifolia in sheep. J Ethnopharmacol. 2004;93(2-3):265-8.

3. Kumar SR, Loveleena D, Godwin S. Medicinal Property of Murraya koenigii A Review. IntRes JBiol Sci.2013;2(9)80-3.

4. Sharma US, Sharma UK, Singh A, SutarN, Singh J.In vitroanthelmintic activity of Murraya koenigii Linn. Leaves extracts. Int J Pharma Bio Sci. 2010;1(3):1-4.

5. Kumar A, TripathiA, Dora J, Tripathi R.Anthelmintic activity of methanolic extract of Murraya koenigii leaves (Linn). Int J Res Pharmaceut Biomed Sci. 2011;2(4):1698-1700.

6. Harborne JB. Phytochemical Methods: A guide for modern techniques of plant analysis. Chapmann and Hall London. 1998;198.

7. Soetan KO, Lasisi OT,Agboluaje AK.Comparative assessment of in-vitro anthelmintic effects of the aqueous extracts of the seeds and leaves of the African locust bean (Parkiabiglobosa) on bovine nematode eggs. J Cell AnimalBiol. 2011;5(6):109-12.

8. Rahman WA,LeeR, SulaimanSF In vitro Anthelmintic activity of Neem Plant (Azadirachtaindica) extracts against third stage Haemonchuscontortus larvae from goats. Global Veterinaria. 2011;7(1):22-6.

9. OECD. Acute oral toxicity test method. In: OECD Guidelines for Testing of Chemicals. No. 420. Paris, France: Organization for Economic Cooperation and Development. 2001

10. Asase A, Oteng-Yeboah AA, OdamttenGT, SimmondsMSJ. Ethnobotanical study of someGhanian anti-malarial plants. J Ethnopharmacol. 2005;99(2):273-9.

11. Priya MN, Darsana U, SreedeviR, Deepa CK, Sujith S. In vitro ovicidal activity of Allophyllus cobbe leaf extract against Haemochus contortus. Int J Applied Pure Sci Agri. 2015;1(3):24-8

12. Priya MN, Sreeshitha SG, Sreedevi R, Sujith S, Deepa CK, Suja RS, et al. Anthelmintic activity of different extracts of Mallotus phillipensis in vitro. Life Sci Int Res J. 2014;1(1):152-5

13. Jeyathilakan N, Murali K, Anandaraj A, Basith SA. Anthelmintic activity of 
essential oils of Cymbopogon citrates and Ocimum sanctum on Fasciolagigantica. J Vet Parasitol. 2010;24:151-4

14. Athanasiadou S, Kyriazaki, Jackson F, CoopRL. Direct anthelmintic effects of condensed tannins towards different gastrointestinal nematodes of sheep: in vitro and in vivo studies. Vet Parasitol. 2001;99(3):205-19.

15. Herve' H, Jackson F, Athanasiadou S, Stig M, Thamsborg. Hoskin SO. The effects of tannin-rich plants on parasitic nematodes in ruminants. Trends in Parasitol. 2006;22(6):253-61.

16. Cenci FB, Louvandini H, McManusCM, Dell'Porto A, Costa DM, Arau'jo SC, et al. Effects of condensed tannin from Acacia mearnsii on sheep infected naturally with gastrointestinal helminthes. Vet Parasitol. 2007;144(1-2):132-7.

17. MinBR, Pomroy WE, HartSP, Sahlu T. The effect of short-term consumption of a forage containing condensed tannins on gastro-intestinal nematode parasite infections in grazing wether goats. Small Ruminant Research. 2004;51(3):279-83.

18. Hernandez-Villegas MM, Borges-Argaez R, Rodriguez-Vivas RI, Torres-Acosta JFJ, Merndez-Gonzalez M, et al. Ovicidal and larvicidal activity of the crude extracts from Phytolacca icosandra against Haemonchus contortus. Vet Parasitol. 2011;179(1-3):100-6.

19. Ademola IO, Fagbemi BO,Idowu SO. Evaluation of the anthelmintic activity of Khaya senegalensisextract against gastrointestinal nematodes of sheep: in vitro and in vivo studies. Vet Parasitol. 2004:122(2):151-64

20. Azando EVB, Hounzangbe-Adote MS, Olounlade PA, Brunet S, Fabre N. Involvement of tannins and flavonoids in the in vitro effects of Newbouldia laevis a and zanthoxylum zanthoxyloides extracts on the exsheathment of third stage infective larvae of gastrointestinal nematodes. Vet Parasitol. 2011;180(3-4):292-7.

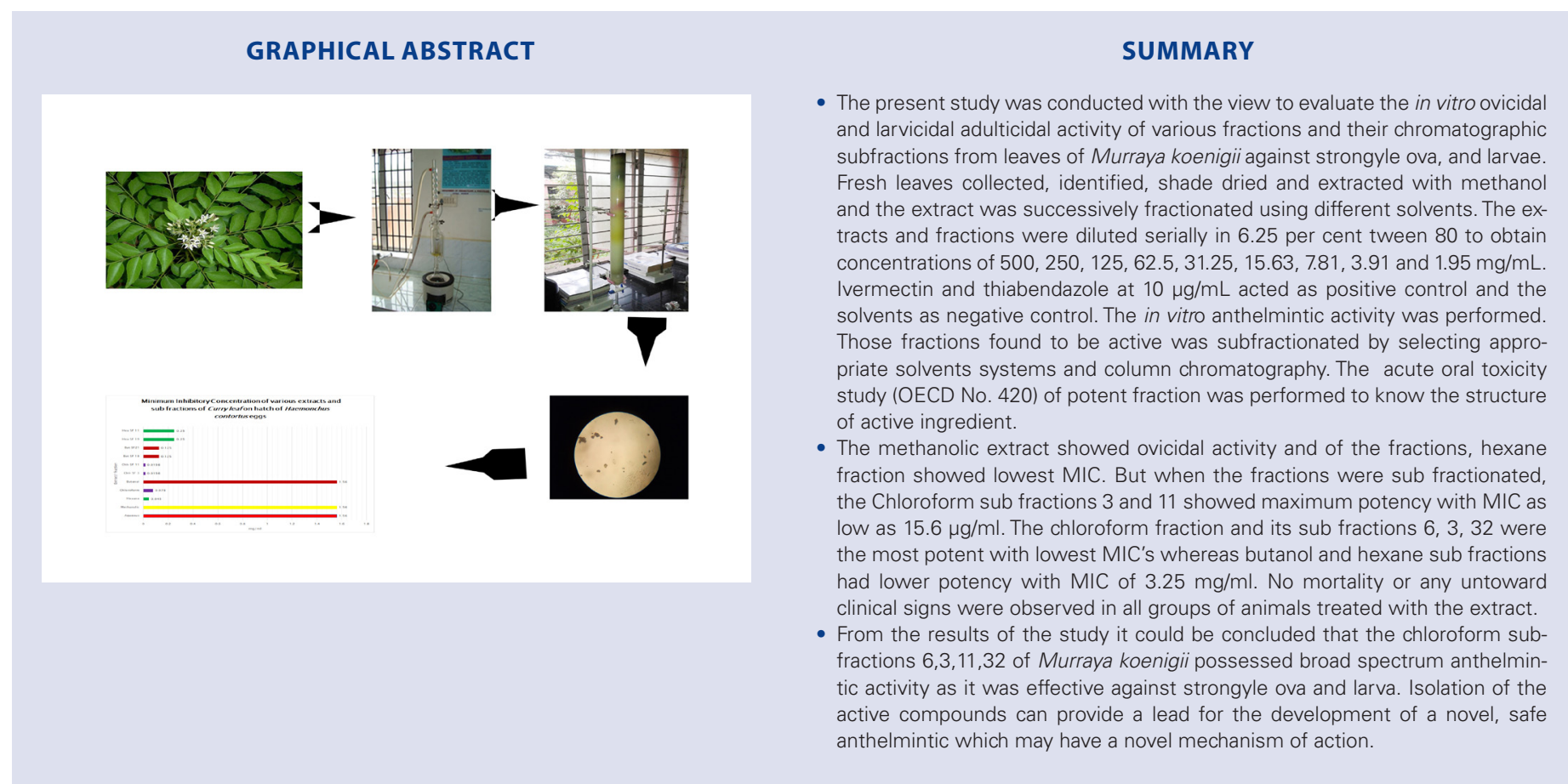

Cite this article: Sujith S, Priya MN, Deepa CK, Usha PTA. Characterizationof the Anthelmintic Activity of Murraya koenigii (Linn.). Pharmacog J. 2018;10(6)Suppl:s100-s103. 